\title{
Generalized analytic functions of Bogdan Ziemian
}

\author{
by GrZegorz Łysik (Warszawa)
}

\begin{abstract}
Definitions, properties, examples and applications of generalized analytic functions introduced by B. Ziemian are presented.

1. Introduction. In Fourier analysis there arises the problem of describing analyticity of a function or distribution in terms of its Fourier transform. The difficulty of the problem is caused by the lack of analytic functions with compact support, or equivalently, the lack of entire functions of exponential growth on $\mathbb{C}^{n}$ which decrease exponentially on $\mathbb{R}^{n}$.

The problem was solved by Bross and Iagolnitzer in [BI] by modifying the Fourier kernel by a quadratic term, which leads to the so-called FBI transformation. On the other hand, Hörmander solved this problem in $[\mathrm{H}]$ by approximating an analytic function by a sequence of smooth functions with a good control of their Fourier transforms. Also Sato solved it in the language of the theory of hyperfunctions ([SKK]).

The approach of Bogdan Ziemian to this problem is based on the Mellin transformation. His idea is quite simple and led him to the definition of a generalized analytic function (GAF). The theory of GAFs was systematically developed in [Z] (written in 1992) in the broad context of functional and complex analysis and its relations to the theory of resurgent functions. It appears that many important special functions as well as solutions to some classes of linear singular ordinary and partial differential equations are GAFs.
\end{abstract}

2. The Mellin transformation and generalized analytic functions. Let us recall the definition of the Mellin transformation. Let $u$ be a measurable function on $\mathbb{R}_{+}$. We define

2000 Mathematics Subject Classification: 34A20, 34A30, 44A15.

Key words and phrases: Mellin transformation, Laplace distributions, special functions, singular differential operators. 


$$
\mathcal{M} u(z)=\int_{0}^{\infty} u(x) x^{-z-1} d x .
$$

To make this integral convergent we assume that $\operatorname{supp} u$ is contained in $I=(0, t]$ with some $t<\infty$, and $u$ is of polynomial growth at zero, i.e. $|u(x)| \leq C x^{\omega}$ for $x>0$ with some $\omega \in \mathbb{R}, C<\infty$. Then $\mathcal{M} u$ defined by (1) is holomorphic on $\{\operatorname{Re} z<\omega\}$; we also denote by $\mathcal{M} u$ its holomorphic extension. The Mellin transformation can be extended to a class of Mellin distributions $M_{(\omega)}^{\prime}(I)$ supported by $I$ (cf. [SZ]). Since $\mathcal{M}(x d u / d x)(z)=z \mathcal{M} u(z)$ (where $d u / d x$ is the distributional derivative of $u$ ) the Mellin transformation is well adapted to the study of differential equations with a regular singular point at zero.

Let $u$ be a smooth function on $\bar{I}=[0, t]$. Then we can write the Taylor formula of any order $N \in \mathbb{N}$,

$$
u(x)=\sum_{j=0}^{N} \frac{u^{(j)}(0)}{j !} x^{j}+x^{N+1} R_{N}(x) \quad \text { for } x \in I
$$

with $R_{N}$ bounded on $I$. Since

$$
\mathcal{M}\left(\chi_{t} x^{j}\right)(z)=\frac{t^{j-z}}{j-z} \quad \text { for } z \neq j
$$

where $\chi_{t}$ denotes the characteristic function of $(0, t]$, we derive that $\mathcal{M}\left(\chi_{t} u\right)$ is holomorphic on $\mathbb{C} \backslash \mathbb{N}_{0}$ with simple poles in $\mathbb{N}_{0}$.

Now assume that $u$ is analytic at zero with radius of convergence greater than $t$. Then the series

$$
\sum_{j=0}^{\infty} \frac{u^{(j)}(0)}{j !} \frac{t^{j-z}}{j-z}
$$

defines a function $G=\mathcal{M}\left(\chi_{t} u\right)$ holomorphic on $\mathbb{C} \backslash \mathbb{N}_{0}$ with simple poles in $\mathbb{N}_{0}$ which satisfies, for any $0<\varepsilon \leq 1$,

$$
|G(z)| \leq \frac{C}{\varepsilon} \cdot \frac{t^{-\operatorname{Re} z}}{1+|\operatorname{Im} z|} \quad \text { for } \operatorname{dist}\left(z, \mathbb{N}_{0}\right) \geq \varepsilon
$$

B. Ziemian observed that the above properties of $G$ completely characterize the Mellin transform of a function $u$ analytic at zero. Namely, if $G=\mathcal{M}\left(\chi_{t} \widetilde{u}\right)$ for some $\widetilde{u}$ continuous on $(0, t]$ of polynomial growth at zero, and $G$ is holomorphic on $\mathbb{C} \backslash \mathbb{N}_{0}$ and satisfies (2) with $C$ independent of $0<\varepsilon \leq 1$ then $\widetilde{u}$ coincides on $(0, t)$ with a function $u(x)=\sum_{j=0}^{\infty} a_{j} x^{j}$ analytic on $|x|<t$. The coefficients $a_{j}, j \in \mathbb{N}_{0}$, are given by the residues of $G$ at $j \in \mathbb{N}_{0}$.

The above observation led B. Ziemian to a generalization of the notion of an analytic function. 
Definition 1 ([SZ], Def. 13.2, [Z], Sec. 11). Let $Z$ be a closed subset of $\omega+\overline{\mathbb{R}}_{+}$for some $\omega \in \mathbb{R}$. A function $u$ continuous on $(0, \varrho), \varrho>0$, and of polynomial growth at zero is called a generalized analytic function of type $Z$ and with radius of convergence $\geq \varrho$ if for any $0<t<\varrho$,

(i) $\mathcal{M}\left(\chi_{t} u\right)$ extends to a holomorphic function on $\mathbb{C} \backslash Z$,

(ii) there exists $s \in \mathbb{R}$ such that for any $\varepsilon>0$ and $\kappa>0$,

$$
\left|\mathcal{M}\left(\chi_{t} u\right)(z)\right| \leq \begin{cases}C(1+|\operatorname{Im} z|)^{s}\left(t e^{\kappa}\right)^{-\operatorname{Re} z} & \text { for } \operatorname{Re} z<0, \quad z \notin Z_{\varepsilon}, \\ C(1+|\operatorname{Im} z|)^{s}\left(t e^{-\kappa}\right)^{-\operatorname{Re} z} & \text { for } \operatorname{Re} z \geq 0, \quad z \notin Z_{\varepsilon},\end{cases}
$$

where $Z_{\varepsilon}:=\{z \in \mathbb{C}: \operatorname{dist}(z, Z) \leq \varepsilon\}$,

(iii) for some $\varepsilon>0$ and any $\kappa>0$ there exists $p=p(\kappa) \in \mathbb{N}$ such that

$$
\left|\mathcal{M}\left(\chi_{t} u\right)(z)\right| \leq C|\operatorname{Im} z|^{-p}\left(t e^{-\kappa}\right)^{-\operatorname{Re} z} \quad \text { for } z \in Z_{\varepsilon}, \operatorname{Im} z \neq 0 .
$$

In fact, generalized analytic functions of type $\mathbb{N}_{0}$ for which (iii) holds with $p=1$ independent of $\kappa>0$ are exactly analytic functions.

Generalized analytic functions can be defined equivalently by means of the space of Laplace distributions $L_{(\log \varrho)}^{\prime}\left(\omega+\overline{\mathbb{R}}_{+}\right), \omega \in \mathbb{R}$ (cf. [Z], Sec. 4).

Definition 1' ([Z], Def. 9.1, [SZ], Th. 13.4). A function $u$ on $(0, \varrho)$ is called a generalized analytic function of type $Z$ with radius of convergence $\varrho$ if there exists a Laplace distribution $S \in L_{(\log \varrho)}^{\prime}\left(\omega+\overline{\mathbb{R}}_{+}\right)$with $\operatorname{supp} S \subset Z$ such that $u$ extends to a function $\widetilde{u}$ holomorphic on the universal covering space $\widetilde{B}(\varrho)$ of the punctured disc $B(\varrho) \backslash\{0\}$ given by

$$
\widetilde{u}(x)=S\left[x^{\cdot}\right] \quad \text { for } x \in \widetilde{B}(\varrho) .
$$

The Laplace distribution $S$ satisfying (3) is called the Borel transform of $u$.

Let $S \in L_{(\log \varrho)}^{\prime}\left(\omega+\overline{\mathbb{R}}_{+}\right)$. Then for any $\kappa>0, S=P_{\kappa}(d / d \alpha) S_{\kappa}$ where $P_{\kappa}$ is a polynomial and $S_{\kappa} \in C^{0}(\mathbb{R})$ with $\operatorname{supp} S_{\kappa} \subset \omega+\overline{\mathbb{R}}_{+}$satisfies $\left|S_{\kappa}(\alpha)\right| \leq$ $C e^{\kappa \alpha} \varrho^{-\alpha}$ for $\alpha \in \omega+\overline{\mathbb{R}}_{+}$. Thus, (3) can be written in the form

$$
\widetilde{u}(x)=P_{\kappa}(-\log x) \int_{\omega}^{\infty} x^{\alpha} S_{\kappa}(\alpha) d \alpha \quad \text { for } 0<|x|<e^{-\kappa} \varrho .
$$

Generalized analytic functions can also be characterized as follows.

TheOREM $1([\mathrm{E}])$. A function $u$ analytic on $(0, \varrho)$ is a GAF of type $\omega+\overline{\mathbb{R}}_{+}$ with radius of convergence $\varrho$ if and only if $u$ extends holomorphically to a function $\widetilde{u} \in \mathcal{O}(\widetilde{B}(\varrho))$ satisfying: for any $\kappa>0$ there exist $C<\infty$ and $m_{\kappa} \in \mathbb{N}_{0}$ such that

$$
|\widetilde{u}(x)| \leq C(1+|\log x|)^{m_{\kappa}}|x|^{\omega} \quad \text { for } 0<|x| \leq e^{-\kappa} \varrho .
$$

The GAFs need not be analytic nor defined at zero, but they have the following quasi-analyticity property. 
Property $A$ ([SZ], $\S 13,[\mathrm{Z}]$, Sec. 9). If $u$ defined on $(0, \varrho), \varrho>0$, is flat at zero of arbitrary order $m \in \mathbb{N}$ (i.e. $u \in O\left(x^{m}\right)$ for any $m \in \mathbb{N}$ ) then $u \equiv 0$.

The GAFs behave well under basic algebraic and differential operations.

Multiplication. If $u_{j}(x)=S_{j}\left[x^{\alpha}\right]$ for $j=1,2$ then $u_{1}(x) \cdot u_{2}(x)=$ $\left(S_{1} * S_{2}\right)\left[x^{\alpha}\right]$ (the convolution is well defined since the support of each $S_{j}$ is contained in a half line). The above property enables one to define nonlinear operations on GAFs.

Differentiation. Let $u(x)=S\left[x^{\alpha}\right]$ with some $S \in L_{(\log \varrho)}^{\prime}\left(\omega+\overline{\mathbb{R}}_{+}\right)$. Then $(d / d x) u, x(d / d x) u$ are GAFs and

$$
\frac{d}{d x} u(x)=\left(\alpha\left(S * \delta_{(-1)}\right)\right)\left[x^{\alpha}\right], \quad x \frac{d}{d x} u(x)=\alpha S\left[x^{\alpha}\right] .
$$

More generally, for any polynomial $P$,

$$
P\left(x \frac{d}{d x}\right) u(x)=P(\alpha) S\left[x^{\alpha}\right] .
$$

Analytic change of variables. Let $g$ be a function analytic in a neighbourhood of zero such that $g(0)=0, g^{\prime}(0) \neq 0$ and let $u$ be continuous on $(0, \varrho)$. Then $u$ is a GAF iff $u \circ g$ is a GAF. The formula expressing the Borel transform of $u \circ g$ in terms of that of $u$ and the derivatives of $g^{-1}$ at zero is more involved and can be found in [Z], Sec. 13.5.

The class of resurgent functions of J. Ecalle ([E]) constitutes an important subspace of GAFs ([Z], Sec. 14). The class consists of those GAFs whose Borel transforms continue analytically to multivalued functions on $\mathbb{C} \backslash \Sigma$ with a discrete set $\Sigma$ of branching points. The operations of taking jumps at the branching points lead to a definition of alien derivatives of resurgent functions which turned out to be a powerful tool in many analytical problems.

\section{Examples}

1. If $S=\delta_{(v)}^{(m)}$ with $v \in \mathbb{R}, m \in \mathbb{N}_{0}$ then

$$
u(x):=S\left[x^{\alpha}\right]=(-1)^{m} \delta_{(v)}\left[D_{\alpha}^{m}\left(x^{\alpha}\right)\right]=(-\log x)^{m} x^{v} .
$$

Thus, a polyhomogeneous function $\sum_{k=0}^{m} \sum_{j=0}^{\infty} a_{k j} x_{+}^{v+j} \log ^{k} x_{+}$is a GAF (if the series are convergent).

2. If $S=(1 / \Gamma(a)) \alpha_{+}^{a-1}$ with $a>0$ ( $\Gamma$ being the Euler function) then

$$
u(x):=S\left[x^{\alpha}\right]=\frac{1}{\Gamma(a)} \int_{0}^{\infty} \alpha^{a-1} x^{\alpha} d \alpha=\frac{1}{(-\log x)^{a}} \quad \text { for } 0<|x|<1 .
$$


3. If $S=(-1 / \alpha) \chi_{[1, \infty)}$ then

$$
u(x):=S\left[x^{\alpha}\right]=-\int_{1}^{\infty} \frac{x^{\alpha}}{\alpha} d \alpha=\int_{0}^{x} \frac{d t}{\log t}=\operatorname{Li}(x) \quad \text { for } 0<|x|<1
$$

is the logarithmic integral. Also other special functions can be considered (after a suitable change of variable) as generalized analytic functions (cf. [Z], Sec. 15).

4. For the hypergeometric function ${ }_{1} F_{1}$ we have

${ }_{1} F_{1}(a, c ;-\log x)=\frac{\Gamma(c)}{\Gamma(c-a) \Gamma(a)} \int_{-1}^{0} x^{\alpha}(-\alpha)^{a-1}(\alpha+1)^{c-a-1} d \alpha \quad$ for $x \in \widetilde{\mathbb{C}}$ where $a>0, c-a>0$.

5. The space of resurgent functions with the set of branching points $\Sigma=\mathbb{N}_{0}$ for which all alien derivatives vanish consists of functions $u$ of the form

$$
u(x)=\sum_{k=0}^{m} c_{k}(\log x)^{k}+\sum_{j=0}^{\infty} \frac{a_{j}}{(-\log x)^{j+1}} \quad \text { for } x \in \widetilde{B}\left(e^{-\omega}\right), \omega>0,
$$

where the series $\sum_{j=0}^{\infty} a_{j} \zeta^{j}$ has radius of convergence $\geq 1 / \omega([\mathrm{Z}]$, Lemma 14.1).

4. Applications. Generalized analytic functions play an important role in the study of differential equations, especially with irregular singular point. To show this observe that the operator $R\left(x, x^{2} d / d x\right)$ under the change of variable $s=e^{-1 / x}$ is transformed into $R(-1 / \log s, s d / d s)$. Thus, we have obtained an operator with a regular singular point at zero but with generalized analytic coefficients. B. Ziemian initiated the study of such operators in Section 16 of [Z]. To formulate the main theorem of that section consider an operator $P$ of the form

$$
R\left(x, x \frac{d}{d x}\right)=P\left(x \frac{d}{d x}\right)+Q\left(x, x \frac{d}{d x}\right)
$$

where $P$ is a polynomial of degree $m \in \mathbb{N}$ and

$$
Q\left(x, x \frac{d}{d x}\right)=\sum_{\nu=0}^{m} a_{\nu}(x)\left(x \frac{d}{d x}\right)^{\nu}
$$

where $a_{\nu}$ are GAFs of convergence radius $\varrho>0$ of the form

$$
a_{\nu}(x)=\int_{\mathbb{R}_{+}} x^{\alpha} \alpha^{k} q_{\nu}(\alpha) d \alpha
$$


with some $k \in \mathbb{N}_{0}$ and $q_{\nu}$ holomorphic in a tubular neighbourhood $V$ of $\mathbb{R}_{+}$ and satisfying, for any $\kappa>0$,

$$
\left|q_{\nu}(\alpha)\right| \leq C_{\kappa} e^{\kappa \alpha} \varrho^{-\alpha} \quad \text { for } \alpha \in V .
$$

For a closed subset $Z$ of $\overline{\mathbb{R}}_{+}$set

$$
Z^{P}=(Z \cup \text { Char } P)+\overline{\mathbb{R}}_{+} \quad \text { where } \quad \text { Char } P=\{z \in \mathbb{C}: P(z)=0\} .
$$

TheOREM 2 ([Z], Theorem 16.2). Under the above notation suppose that the maximal multiplicity of the roots of $P$ is not greater than $k+1$ and $f$ is a generalized analytic function of type $Z$. Then there exists $r>0$ such that any solution of $R u=f$ on $(0, \varrho)$ is a $G A F$ of type $Z^{P}$ and convergence radius not less than $r$.

The definition of GAFs extends to the $n$-dimensional case by taking for $S$ a Laplace distribution supported by the $n$-dimensional positive "octant" $\overline{\mathbb{R}}_{+}^{n}$ or more generally its image under a holomorphic mapping (cf. [Z], Sec. 18).

Finally, let us quote Theorem 19.1 from [Z], which shows an application of GAFs in PDEs.

Theorem 3. Let $R(x, x \partial / \partial x)=P(x \partial / \partial x)+Q(x, x \partial / \partial x)$ be a Fuchsian type partial differential operator, where $P$ is an elliptic operator of order $m$ and $Q(x, x \partial / \partial x)=\sum_{|\nu| \leq m} a_{\nu}(x)(x \partial / \partial x)^{\nu}$ is a linear operator with coefficients $a_{\nu},|\nu| \leq m$, of the form

$$
a_{\nu}(x)=\int_{\mathbb{R}_{+}^{n}} x^{\alpha} \alpha^{\mathbf{k}} q_{\nu}(\alpha) d \alpha
$$

with some $\mathbf{k}=(k, \ldots, k) \in \mathbb{N}_{0}^{n}$ and $q_{\nu}$ for $|\nu| \leq m$ holomorphically extendable to functions of exponential growth on a polytubular neighbourhood of $\overline{\mathbb{R}}_{+}^{n}$. Assume that $P$ satisfies the cone condition with respect to convex open cones $\Gamma \subset \operatorname{Im} \mathbb{C}^{n}$ and $-\Gamma$, i.e. $\|\operatorname{Im} z\|^{p} /|P(z)|$ is bounded for $\operatorname{Im} z \in \pm \Gamma$, $\operatorname{Im} z$ small, with some $p \in \mathbb{N}$. If $k+1 \geq p$ then for any generalized analytic function $f$ (in $n$ variables) there exists $r>0$ and a generalized analytic function $u$ which solves the equation

$$
R(x, x \partial / \partial x) u=f
$$

in the polyinterval $\{0<x<(r, \ldots, r)\}$.

\section{References}

[BI] J. Bros and D. Iagolnitzer, Causality and local analyticity; mathematical study, Ann. Inst. H. Poincaré 18 (1973), 147-184.

[E] J. Ecalle, Les fonctions résurgentes, Publ. Math. Univ. Paris-Sud, several volumes. 
[H] L. Hörmander, The Analysis of Linear Partial Differential Operators I, Springer, 1983.

[€] G. Eysik, Generalized analytic functions and a strong quasi-analyticity principle, Dissertationes Math. 340 (1995), 195-200.

[SKK] M. Sato, T. Kawai and M. Kashiwara, Hyperfunctions and pseudodifferential equations, Lecture Notes in Math. 287, Springer, 1973, 263-529.

[SZ] Z. Szmydt and B. Ziemian, The Mellin Transformation and Fuchsian Type Partial Differential Equations, Kluwer, 1992.

[Z] B. Ziemian, Generalized analytic functions with applications to singular ordinary and partial differential equations, Dissertationes Math. 354 (1996).

Institute of Mathematics

Polish Academy of Sciences

P.O. Box 137

00-950 Warszawa, Poland

E-mail: lysik@impan.gov.pl 